\title{
AIR CONDITIONING SYSTEM DESIGN AND STRATEGIES FOR IMPROVING ENERGY EFFICIENCY - A REVIEW
}

\section{AMINA ISMAIL \& FREDDIE INAMBAO}

Department of Mechanical Engineering, University of Kwazulu-Natal, Durban, South Africa

\section{ABSTRACT}

Centrifugal water-cooled chiller systems consume approximately 50\% of a building's total energy and are an essential component providing indoor environmental quality (IEQ) and enhancing occupant productivity in large scale buildings. Therefore, research into reducing energy consumption required for air conditioning while maintaining the desired level of IEQ has been stimulated. The relationship between thermal energy and electrical energy in this system can be used to reduce the energy consumption of the system which will involve the overall design and operating parameters of rotating equipment, static equipment for heat transfer as well as how this equipment operate in relation to each other. This review provides a holistic approach to chiller system design and the potential it has to improve energy efficiency of the system. Based on the research, energy efficient equipment selection, the use of new technology, energy efficient design techniques/practices and the use of control systems will optimize the energy consumption of the HVAC system.

KEYWORDS: Centrifugal Water-Cooled Chiller, Energy Efficiency, Thermal Energy, Electrical Energy \& Energy Efficient Technology

Received: Mar 03, 2021; Accepted: Mar 23, 2021; Published: May 11, 2021; Paper Id.: IJMPERDJUN202126

\section{INTRODUCTION}

In 2018 the world energy demand grew by $2.3 \%$ which is the fastest over the last decade, with fossil fuels constituting 70\% of this growth [1], people spend approximately $90 \%$ of their time indoors [2], and there is a growing reliance on building thermal comfort especially when compared to acoustics and visual comfort [3]. Heating, ventilation and air conditioning (HVAC) systems form an essential component in the built environment as buildings are becoming more advanced and require a certain level of thermal comfort for occupant satisfaction as well as a high level of employee productivity. Therefore, the development of HVAC systems that utilise renewable energy sources with energy efficient primary and secondary system equipment will reduce the total energy consumption of a building [4]. Looking at HVAC energy consumption per building worldwide, the United States (US) accounts for more than $48 \%$ of a building's energy consumption [5], Spain and Italy more than 50\% [5] and Australia about $70 \%$ [4]. In the Middle East, more than $70 \%$ of a building's energy consumption is for indoor thermal comfort [6]. On an average, HVAC systems are known to consume approximately $50 \%$ of the total energy demand of a commercial building [7], [8]. According to [9] and [10], in South Africa, HVAC systems consume between $30 \%$ to $50 \%$ of a commercial building's total energy consumption. To alleviate this challenge, it is essential to develop energy efficient HVAC systems and utilise renewable energy as the main source of electrical energy supply. Energy efficient HVAC systems also enhance the value of a property, protect property owners from increasing energy costs and contribute to the protection of the environment from harmful greenhouse gas (GHG) emissions. 
With advances in science and technology, there are several ways to achieve energy efficient HVAC systems for commercial buildings. However, to successfully design the system, it is important to know and understand the function of the building, the indoor thermal comfort requirements of the occupants, and the climatic conditions of that location. Research indicates that energy efficiency and thermal comfort can be achieved by a well-designed air-conditioning system and an appropriate selection of air conditioning equipment and control strategies to reduce energy consumption and enhance indoor environmental quality (IEQ) [4].

Chiller plants are widely used throughout South African government buildings because they are most suitable, considering the climatic conditions and thermal comfort requirements as well as the maintenance of chillers. The HVAC industry is extensive; therefore, this research will focus on reviewing recent novel technologies and strategies related to optimizing the energy efficiency of centrifugal water-cooled chillers. Referring to Error! Reference source not found., this article will present the main energy consuming components of the water-cooled chiller and energy losses in the system followed by new technologies that enhance the performance of energy efficiency. Thereafter, a review of innovative chiller systems designs to improve energy consumption will be discussed. Lastly, recent developments in chiller control strategies and smart chiller sequencing methodologies that enhance energy performance of the chillers will be explored.

\section{ENERGY EFFICIENT TECHNOLOGY}

The major components of the centrifugal water-cooled chillers are the compressor, the cooling tower, the pumps (with reference to the chilled water pumps and the cooling water pumps) and the fans in the air handling unit. Essentially, each component contributes to the efficiency of the whole system, therefore a wholistic approach is important in achieving a low energy input per unit of cooling. This section intends to provide a breakdown of recent technologies that can lower the $\mathrm{kW} /$ ton of cooling in a water-cooled centrifugal chiller.

\subsection{Compressor}

The design of a centrifugal compressor plays an important role in improving its efficiency and contributing to the overall efficiency of the air conditioning system. Each component of the centrifugal compressor contributes to the optimization of the compressor. By improving the efficiency of the components, overall efficiency of the compressor can be achieved. In 2009, Timaki et al carried out a computational fluid dynamics (CFD) analysis to develop a high efficiency centrifugal compressor for a turbo chiller [1]. A 1000 ton turbo chiller with a coefficient of performance (COP) of 6.0 was used as a baseline against which the performance of the chiller was gauged [1]. The authors reduced the thickness and increased the number of the compressor blades for stage 1 and stage 2 [1]. The length of the splitter blades on the shroud side was increased. The impeller outlet angle was increased to increase the stable operating range of the $1^{\text {st }}$ impeller stage [1]. The shape of the leading edges of the $1^{\text {st }}$ and $2^{\text {nd }}$ stage impellers were modified from an arc to an ellipse to improve the flow pattern inside the impellers [1]. The splitter blade shape was also modified to smooth out the flow between the splitter blades and the full blades [1]. Timaki et al. concluded that the new design of the turbo compressor improved the COP of chiller capacities ranging to 700 tons of refrigerant to a COP of 6.3 [1]. According to [2], variable speed centrifugal chillers perform more efficiently at part load ratio of the cooling demand and at partial compression ratio of lift head when compared to constant speed centrifugal chillers. The benefits of variable speed drives are further improved with magnetic bearing technology [2]. The overall results show that optimal chiller staging only improves the system performance by 
$1.2 \%$ for a constant flow system. However, using a VSD with magnetic bearings increased the energy savings to $13.7 \%$ $[2]$.

\subsection{Variable Speed Drive}

Systems and processes often have variable flows or operate within certain parameters when accommodated by the conventional constant speed motors that provide a constant output. To improve the efficiency and accuracy of the operation, modern technology has advanced, thus, developing the variable speed drive (VSD). A VSD is a device that regulates the rotational speed and force of a motor according to the required output. VSDs are used to drive pumps, fans, compressors, and other equipment. The use of VSDs have the potential to closely regulate the fluid flow according to the operating conditions and thus reduce unnecessary energy consumption. The research carried out by Al-Bassam and Alasseri found that VSDs are energy efficient as the results from their research indicate that water consumption was reduced by $13 \%$ when compared to the commonly used motors and the chiller and cooling tower power consumption was reduced by $5.8 \%$ [3]. Chuang et al. showed that a stepless VSD combined with a control system can save at least $20 \%$ of the energy consumed [4]. Saidur et al. concluded that VSDs are reliable and cost effective and save energy thus promoting energy efficiency [5]. Saidur et al. also indicated that VSDs not only provide energy efficient capacity control, but they also have a low starting current and reduce the mechanical stresses on motors and belts [5].

\subsection{Thermal Storage Systems}

A thermal storage system (TSS) is a technology that stores thermal energy by either heating or cooling the medium, so the thermal energy can be used for heating or cooling at a later period. A TSS system is able to balance the energy demand between peak and off-peak hours [6]. During off-peak hours, cool energy is usually stored in the form of ice, phase change materials, chilled water, or eutectic solution. This energy is used during peak hours; as the phase of the material changes, heat energy is absorbed providing cooling to the working fluid [6]. Rahman et al. carried out an investigation to determine the feasibility of a TSS and concluded that a TSS can save up to $61.19 \%$ of the electricity cost required for cooling when compared to conventional systems [7]. The research carried out by Saddat-Mohammadi et al. shows that chilled water storage with the use of robust optimization reduces the overall electricity costs and that a TSS is more efficient for systems that have a high cooling load during peak hours [8]. The research carried out by Lin et al. involved analysing two new airconditioning systems with TSS [9]. The results from their research indicated that TSS contributes to financial savings as well as energy savings [9].

\section{SYSTEM DESIGN}

Air conditioning system design plays an important role in energy consumption. Energy efficiency can be enhanced by utilising energy efficient equipment within an energy efficient system. System design can ultimately decide whether energy is being conserved or not. The design engineer should incorporate various techniques and methodologies to ensure that energy efficient measures are implemented.

\subsection{Air-to-Air Heat Exchangers}

One of the primary ways of reducing energy consumption of an HVAC system is to utilise an air-to-air heat exchanger (AAHE). A mechanical heat exchanger unit is used to transfer energy between two streams of air, the exhaust air and the fresh air supply, refer to Figure. The main role of this device is to pre-cool the fresh air supply before entering the AHU, 
thus reducing the amount of energy required to condition the fresh air. Wanli et al. proposed five solutions to enhance energy efficiency of air handling units (AHU) [10]. Their study used AAHE to reduce the cooling and heating loads on the coil [10]. To reduce the AHU power consumption, they analysed the energy and exergy losses of all five AHU layouts against the base design [10]. The energy balance calculations from their investigation revealed that AAHEs affect the power consumption and can reduce the power consumption in the coils [10]. Zhang et al. investigated two scenarios to enhance the performance of conventional evaporative cooling and condensation dehumidification systems and found that the COP of the base system increased by $8.5 \%$ and the COP of the first and second systems increased by $12.09 \%$ [11]. Eades carries out an investigation to examine ways of reducing energy and water consumption in laboratory HVAC systems. The author's model predicts energy savings of up to $13.5 \%$ and shows the efficiency of using AHU condensate for energy recovery purposes [12]. One of the most effective methods of HVAC systems design is thermal energy recovery and transporting energy from one space to another where it is required.

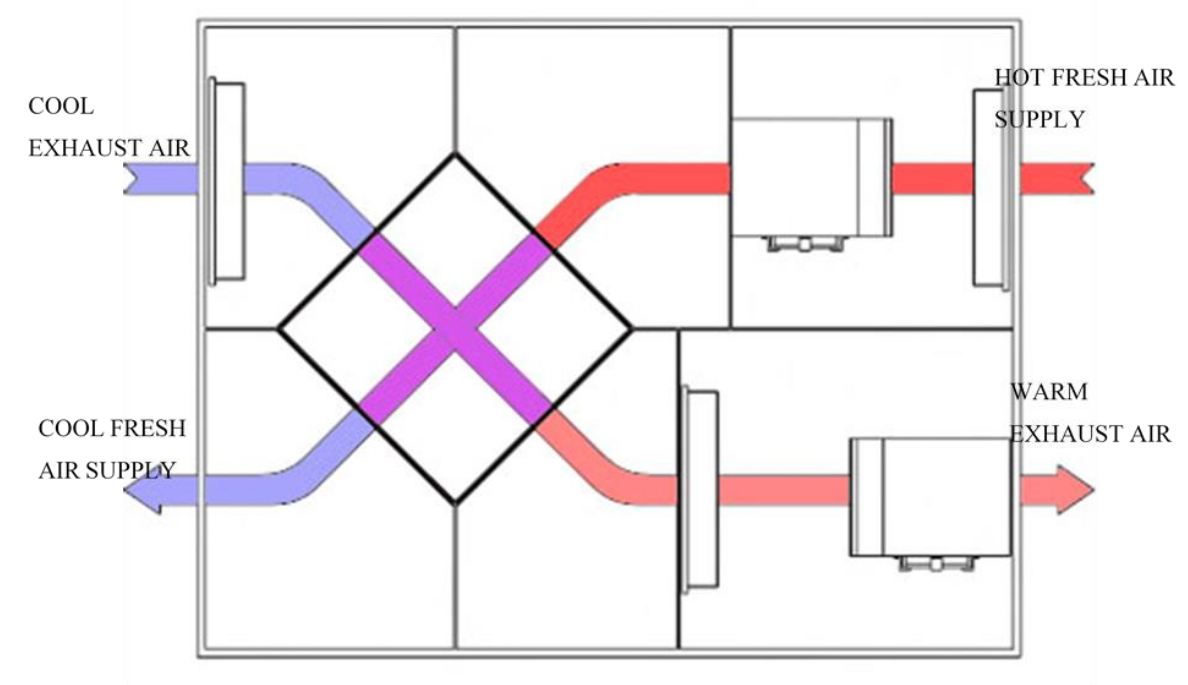

Figure 1: Air-to-Air Heat Exchanger Mixing Box (Image Adapted from [13]).

\subsection{Solar Assisted Air Conditioning}

There are various means of harnessing solar energy with one of the most popular methods being electrical energy produced with photovoltaic panels. This electrical energy is then stored in batteries and then used to power the HVAC system [14]. Another method is the solar thermal method that converts solar energy to heat energy, therefore, heating up the working refrigerant using solar energy and reducing the compressor power input. Figure 1 is a schematic of a solar absorption cooling system used for solar assisted air conditioning [14], [15]. An interesting study was carried out by Aguilar-Jimenez et al. in an off-grid school in Mexico where an absorption cooling system with $35 \mathrm{~kW}$ capacity driven by solar thermal energy was installed [16]. The outcome from the study showed that the cooling system was able to run continuously taking advantage of the stored energy and only utilizing less than $75 \%$ of the cooling capacity [16]. The system effortlessly provided cooling for all four classrooms during a five-day week from 7 am to $3 \mathrm{pm}$ [16]. Hashe reviews the sustainability of solar heating and cooling and concludes that solar energy is a sustainable alternative to reduce the use of fossil fuels [17]. Solar irradiation and air conditioning are quite coincidental where air conditioning energy consumption increases as the heat gain increases, therefore, making solar thermal energy and cooling a favourable option for reducing energy consumption for cooling systems [18]. In 2009, Fong et al conducted a comparative study to investigate which system has 
the greatest energy saving potential [19]. The authors investigated the performance of five solar cooling systems; a) solar electric compression refrigeration, b) solar mechanical compression refrigeration, c) solar absorption refrigeration, d) solar adsorption refrigeration and e) solar solid desiccant cooling [18], [19]. The key finding from Fong's et al investigation indicated that solar electric compression refrigeration and solar absorption refrigeration yielded the highest potential for energy saving [18], [19]. Another investigation was carried out by Zhai and Wang in 2008 wherein five different absorption and adsorption chiller configurations were compared and summarised [20]. One of the outcomes indicated that solar absorption cooling systems are suitable for air conditioning large buildings and solar adsorption cooling systems are suitable for smaller buildings [20]. Their research highly recommends the design of solar powered integrated systems in public buildings [20].

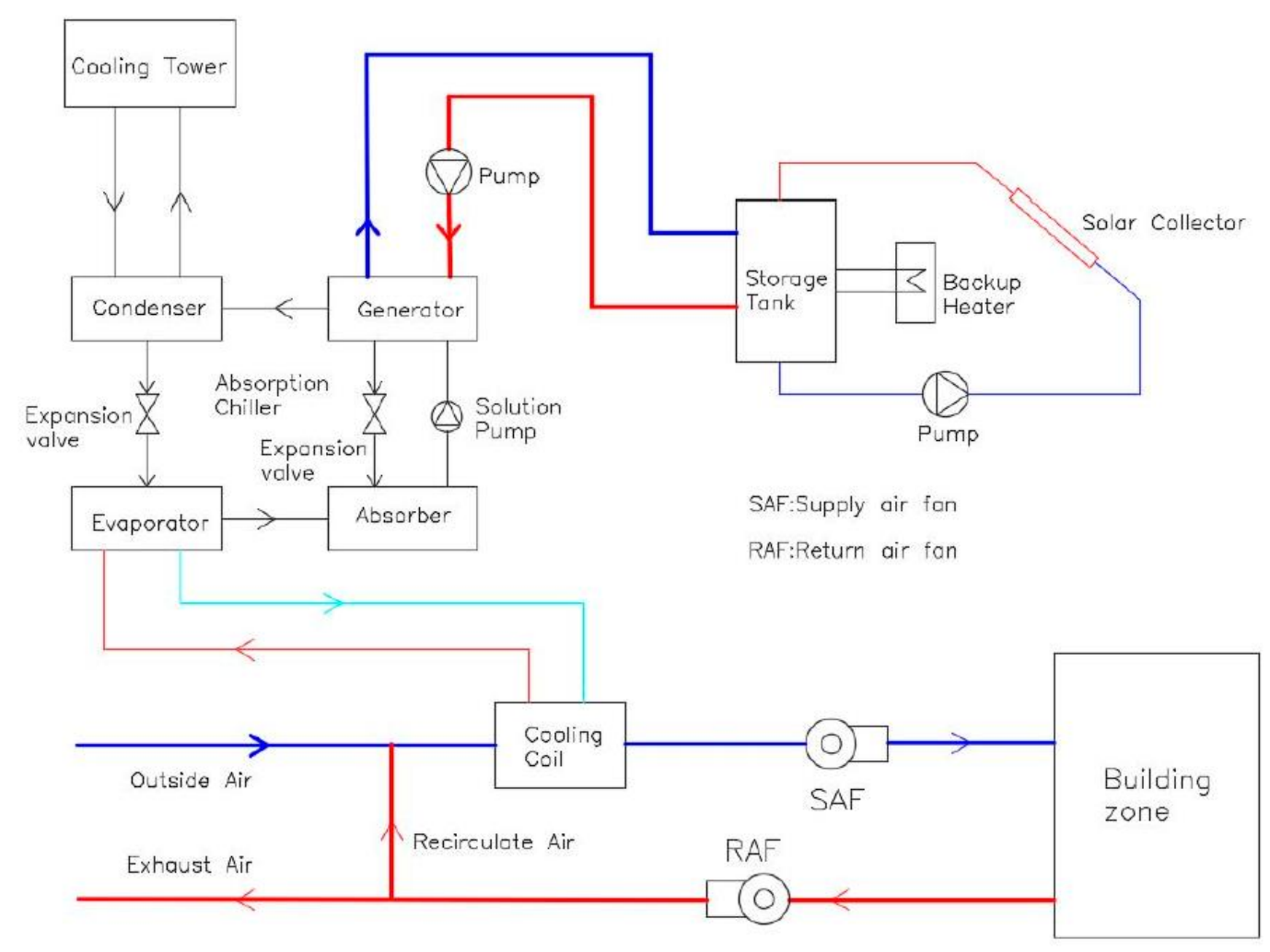

Figure 1: Schematic Diagram of Solar Absorption Cooling System [15].

\section{OPERATIONAL MANAGEMENT AND CONTROL SYSTEM}

The dynamics of the fourth industrial revolution have made the vision of an intelligent air conditioning system achievable. Management and control systems can be used to achieve optimal indoor air quality and environmental conditions and yet keep energy consumption relatively low. A concerted effort is made to study intelligent air flow and control strategies to address improved IAQ and to contribute to the development of sustainable buildings by reducing energy consumption [18]. Currently, there is extensive research in the field of HVAC control systems to develop intelligent air quality, air temperature, humidity, and velocity control. Monitoring and adjusting these IEQ parameters involves adjusting the operating conditions of the compressor, the pumps, fans, and dampers in the system. Developing an intelligent control system for a large-scale air conditioning system involves a holistic view and understanding of the functioning of the system. 
A $\mathrm{CO}_{2}$ based adaptive demand controlled ventilation (DCV) strategy was implemented and monitored in a high rise building in Hong Kong [21]. A dynamic multizone ventilation equation for multi-zone air conditioning systems was used for the adaptive DCV strategy wherein the total number of occupants in the space was detected using the $\mathrm{CO}_{2}$ concentration in the air, this data was then logged onto an online occupancy detection system [21]. It was found that the DCV is able to significantly reduce energy consumption during summer, however, it may not be suitable for outdoor air control in winter [21].

Freire et al. [22] conducted a study on the effects of minimizing energy consumption while maintaining adequate indoor thermal comfort. Predicted mean vote and the comfort zone on the psychometric chart was used to define the occupants comfort levels for this investigation [22]. The methods used are based on model based predictive control (MBPC) and simulation results were presented for two case studies [22]. The control algorithms proposed use only one actuator system that can be associated to a heating or cooling system [22]. The simulation results obtained indicated that the algorithms used can simultaneously reduce energy consumption and promote thermal comfort while remaining $100 \%$ of the time in the comfort zone [22].

Another study proposed a risk based online robust optimal control strategy for multi-zone air conditioning systems [23]. As the core of the strategy, a risk-based online control decision scheme is developed for decision-making by considering the ambiguities in the information, the failure risks and energy benefits of different modes are compromised [23]. The results from this study showed that the proposed strategy made the optimal online control decisions while allowing for any uncertainties [23]. The proposed strategy achieved approximately $20 \%$ overall energy saving in the test period when compared to commonly used control strategies [23].

To achieve distributed optimal control of building HVAC systems, an agent based optimal control strategy was proposed for deployment in smart sensors integrated in internet of things (IoT) based field networks and local controllers in field networks of current local area network (LAN) based building automation systems [24]. The component agents and a coordinator agent are designed to deal with simple tasks assigned to them [24]. The tasks are simplified by applying the dual decomposition to an original complex optimization task [24]. The performance and implementation variables of the proposed agent based optimal control strategy have been assessed by tests on a simulated central cooling plant [24]. One of the key findings of the proposed agent based optimal control strategy is that it can achieve significant energy savings when compared with conventional control strategies [24]. When compared with the near optimal control strategy, savings of $3.36 \%$ and $4.6 \%$ were achieved [24].

Control strategies for large-scale HVAC systems have become more frequently researched. There are various techniques and control strategies that can be adopted to improve the energy consumption of large facilities while maintaining the recommended IAQ and IEQ comfort levels.

\section{DISCUSSIONS}

The three main types of equipment to enhance energy efficiency that need to be considered are the type of compressor, variable speed drives and the thermal storage system. The design of the compressor impellers, the use of magnetic bearings and VSDs; all improve the efficiency of the compressor. VSDs coupled to pumps and fans in the system improve the energy efficiency of these pieces of equipment. A thermal storage system is useful for energy efficiency and financial 
savings.

The design of the air conditioning system has the potential to improve the energy consumption by using various design practices, for example, transferring waste thermal energy to where it can be used. Air-to-air heat exchangers have proven beneficial in reducing the amount of energy required to cool down ventilation air before entering the space. The use of solar thermal panels to assist or run air conditioning systems is another popular consideration that has gained momentum in the research realm and is highly recommended.

Operational management and control systems are proven to enhance the efficiency of HVAC systems. However, there are various control strategies that can be implemented with each strategy having unique advantages and disadvantages. Due to the extent of large-scale air conditioning systems, control strategies are becoming increasingly complex. There are certain levels of control to monitor IEQ parameters and regulate the system accordingly, however, to optimally control a HVAC system to maintain the desired level of IEQ and thermal comfort with minimal human interference is currently a popular topic in research.

\section{CONCLUSIONS}

Chiller systems are an essential component used for air conditioning in commercial buildings consuming approximately $50 \%$ of the buildings total energy. This has stimulated research into reducing the energy consumption required for air conditioning while maintaining the desired level of IEQ with minimal to no human interference. A holistic approach to system design has the potential to improve energy efficiency of the system. This review reveals that equipment selection, the use of new technology, energy efficient design techniques/practices and the use of control systems can optimize the energy consumption of the HVAC system.

\section{REFERENCES}

1. T. Hideaki, P. E. Jp, T. Machinery, and E. Technology, "Development of High-Efficiency Centrifugal Compressor for Turbo Chiller," vol. 42, no. 2, pp. 77-84, 2009.

2. L. Wang, Y. Sakurai, and D. E. Claridge, "Optimize a chilled-water plant with magnetic-bearing variable speed chillers," ASHRAE Trans., vol. 126, pp. 725-735, 2020.

3. E. Al-Bassam and R. Alasseri, "Measurable energy savings of installing variable frequency drives for cooling towers' fans, compared to dual speed motors," Energy Build., vol. 67, pp. 261-266, 2013, doi: 10.1016/j.enbuild.2013.07.081.

4. H. C. Chuang, Y. X. Zeng, and C. T. Lee, "Study on a chiller of air conditioning system by sensing refrigerant pressure feedback control with stepless variable speed driving technology, "Build. Environ., vol. 149, no. December 2018, pp. 157-168, 2019, doi: 10.1016/j.buildenv.2018.12.024.

5. R. Saidur, S. Mekhilef, M. B. Ali, A. Safari, and H. A. Mohammed, "Applications of variable speed drive (VSD) in electrical motors energy savings," Renew. Sustain. Energy Rev., vol. 16, no. 1, pp. 543-550, 2012, doi: 10.1016/j.rser.2011.08.020.

6. Sow, G. Bop D. "STUDY OF THE ENERGY BEHAVIOR OF DIFFERENT REFRIGERANTS: ENERGY COST AND ENVIRONMENTAL IMPACT. International Journal of Applied and Natural Sciences (IJANS)

7. Y. H. Yau and B. Rismanchi, "A review on cool thermal storage technologies and operating strategies," Renew. Sustain. Energy Rev., vol. 16, no. 1, pp. 787-797, 2012, doi: 10.1016/j.rser.2011.09.004.

8. M. M. Rahman, M. G. Rasul, and M. M. K. Khan, "Feasibility of thermal energy storage systems in an institutional building in 
subtropical climates in Australia," Appl. Therm. Eng., vol. 31, no. 14-15, pp. 2943-2950, 2011, doi: 10.1016/j.applthermaleng.2011.05.025.

9. Hassan, Mohammad Ul, and Sudarshan Singh. "Fabrication, Experimentation, Performance Evaluation of Two Stage Air Cooler and Comparison with Conventional Air Cooler." International Journal of Mechanical Engineering (IJME) 5.4 (2016): 75-84.

10. M. Sadat-Mohammadi, S. Asadi, M. Habibnezhad, and H. Jebelli, "Robust scheduling of multi-chiller system with chilledwater storage under hourly electricity pricing, "Energy Build., vol. 218, 2020, doi: 10.1016/j.enbuild.2020.110058.

11. H. Lin, X. H. Li, P. S. Cheng, and B. G. Xu, "Study on chilled energy storage of air-conditioning system with energy saving," Energy Build., vol. 79, pp. 41-46, 2014, doi: 10.1016/j.enbuild.2014.04.047.

12. W. Liu, R. Kalbasi, and M. Afrand, "Solutions for enhancement of energy and exergy efficiencies in air handling units," J. Clean. Prod., vol. 257, 2020, doi: 10.1016/j.jclepro.2020.120565.

13. L. Zhang, X. Zha, X. Song, and X. Zhang, "Optimization analysis of a hybrid fresh air handling system based on evaporative cooling and condensation dehumidification," Energy Convers. Manag., vol. 180, no. October 2018, pp. 83-93, 2019, doi: 10.1016/j.enconman.2018.10.100.

14. W. G. Eades, "Energy and water recovery using air-handling unit condensate from laboratory HVAC systems,” Sustain. Cities Soc., vol. 42, no. April, pp. 162-175, 2018, doi: 10.1016/j.scs.2018.07.006.

15. L. Santalla, “Ventilate with heat recovery units," 2012. http://teoriadeconstruccion.net/blog/ventilar-con-recuperadores-decalor/ (accessed Dec. 01, 2020).

16. O. Bamisile, O. Olagoke, M. Dagbasi, F. Dika, and B. Okwesi, "Review of solar assisted HVAC systems; Its performance analysis using CO2 as a refrigerant," Energy Sources, Part A Recover. Util. Environ. Eff., vol. 41, no. 24, pp. 2957-2974, 2019, doi: 10.1080/15567036.2019.1582736.

17. Y. Ma, S. C. Saha, W. Miller, and L. Guan, "Comparison of different solar-assisted air conditioning systems for Australian office buildings," Energies, vol. 10, no. 10, 2017, doi: 10.3390/en10101463.

18. J. A. Aguilar-Jiménez, N. Velázquez, R. López-Zavala, L. A. González-Uribe, R. Beltrán, and L. Hernández-Callejo, "Simulation of a solar-assisted air-conditioning system applied to a remote school," Appl. Sci., vol. 9, no. 16, 2019, doi: 10.3390/app9163398.

19. V. T. Hashe, "Solar Heating and Cooling in Buildings - How Sustainable?," Procedia Manuf., vol. 7, pp. 92-97, 2017, doi: 10.1016/j.promfg.2016.12.024.

20. K. J. Chua, S. K. Chou, W. M. Yang, and J. Yan, “Achieving better energy-efficient air conditioning - A review of technologies and strategies," Appl. Energy, vol. 104, pp. 87-104, 2013, doi: 10.1016/j.apenergy.2012.10.037.

21. K. F. Fong, T. T. Chow, C. K. Lee, Z. Lin, and L. S. Chan, "Comparative study of different solar cooling systems for buildings in subtropical city," Sol. Energy, vol. 84, no. 2, pp. 227-244, 2010, doi: 10.1016/j.solener.2009.11.002.

22. X. Q. Zhai and R. Z. Wang, “A review for absorbtion and adsorbtion solar cooling systems in China," Renew. Sustain. Energy Rev., vol. 13, no. 6-7, pp. 1523-1531, 2009, doi: 10.1016/j.rser.2008.09.022.

23. Z. Sun, S. Wang, and Z. Ma, "In-situ implementation and validation of a CO2-based adaptive demand-controlled ventilation strategy in a multi-zone office building," Build. Environ., vol. 46, no. 1, pp. 124-133, 2011, doi: 10.1016/j.buildenv.2010.07.008.

24. R. Z. Freire, G. H. C. Oliveira, and N. Mendes, "Predictive controllers for thermal comfort optimization and energy savings," 
Energy Build., vol. 40, no. 7, pp. 1353-1365, 2008, doi: 10.1016/j.enbuild.2007.12.007.

25. C. Zhuang and S. Wang, "Risk-based online robust optimal control of air-conditioning systems for buildings requiring strict humidity control considering measurement uncertainties," Appl. Energy, vol. 261, no. December 2019, p. 114451, 2020, doi: 10.1016/j.apenergy.2019.114451.

26. B. Su and S. Wang, "An agent-based distributed real-time optimal control strategy for building HVAC systems for applications in the context of future IoT-based smart sensor networks," Appl. Energy, vol. 274, no. May, p. 115322, 2020, doi: 10.1016/j.apenergy.2020.115322. 
